\title{
THE SCIENCE
}

\section{THE ART}

\section{THE LEADER WITHIN}

\section{Nurse Manager Competencies}

MAONL

American Organization for Nursing Leadership 
MAONL

Suggested APA Citation: AONE, AONL. (2015). AONL Nurse Manager Competencies. Chicago, IL: AONE, AONL.

Accessed at: www.aonl.org

Accessible at: www.aonl.org/competencies

Contact: aonl@aha.org or (312) 422-2800

(C) 2015 American Organization of Nurse Executives (AONE), American Organization for Nursing Leadership (AONL). All materials contained in this publication are available to anyone for download on www.aonl.org, for personal, non-commercial use only. No part of this publication may be reproduced and distributed in any form without permission of AONL, except in the case of brief quotations followed by the above suggested citation. To request permission to reproduce this material, please email aonl@aha.org. 


\section{OVERVIEW}

N urse managers-nurse leaders with 24 hour accountability and responsibility for a direct care unit or units-provide the vital link between the administrative strategic plan and the point of care. The nurse manager is responsible for creating safe, healthy environments that support the work of the health care team and contribute to patient engagement. The role is influential in creating a professional environment and fostering a culture where interdisciplinary team members are able to contribute to optimal patient outcomes and grow professionally.

The Nurse Manager Competencies are based on the Nurse Manager Learning Domain Framework and capture the skills, knowledge and abilities that guide

The Science:

Managing the Business

Financial Management

Human Resource Management

Performance Improvement

Foundational Thinking Skills

Technology

Strategic Management

Clinical Practice Knowledge
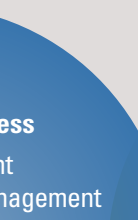

\section{保}

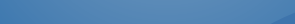

\section{THE NURSE} MANAGER

\section{MANAGER}

The Leader Within: Creating the Leader in Yourself

Personal and Professional Accountability Career Planning Personal Journey Disciplines Optimizing the Leader Within the practice of these nurse leaders. The successful nurse leader must gain expertise in all three domains.

\footnotetext{
In 2004, the American Association of Critical-Care Nurses (AACN) the American Organization for Nursing Leadership (AONL), and the Association of peri-Operative Registered Nurses (AORN) formed the Nurse Manager Leadership Collaborative for the purpose to identify and organize the skills required to perform the job of the nurse manager. In 2006, AONL and AACN formed the Nurse Manager Leadership Partnership (NMLP) to continue this leadership work.

Reliability and validity for the Nurse Manger Competencies is established by periodic job analysis/role delineation studies. These competencies are based on the A National Practice Analysis Study of the Nurse Manager and Leader (2014).
} 


\section{THE SCIENCE}

\section{A. FINANCIAL MANAGEMENT}

1. Recognize the impact of reimbursement on revenue

2. Anticipate the effects of changes on reimbursement programs for patient care

3. Maximize care efficiency and throughput

4. Understand the relationship between value-based purchasing and quality outcomes with revenue and reimbursement

5. Create a budget

6. Monitor a budget

7. Analyze a budget and explain variance

8. Conduct ongoing evaluation of productivity

9. Forecast future revenue and expenses

10. Capital budgeting

» Justification

»Cost benefit analysis

\section{B. HUMAN RESOURCE MANAGEMENT}

1. Staffing needs

»Evaluate staffing patterns/needs

»Match staff competency with patient acuity

2. Manage human resources within the scope of labor laws

3. Apply recruitment techniques

4. Staff selection

»Apply individual interview techniques

"Apply team interview techniques

»Select and hire qualified applicants

5. Scope of practice

»Develop role definitions for staff consistent with scope of practice

»Implement changes in role consistent with scope of practice
»Orientation

"Develop orientation program

"Oversee orientation process

»Evaluate effectiveness of orientation

\section{PERFORMANCE IMPROVEMENT}

1. Performance improvement

»Identify key performance indicators

»Establish data collection methodology

»Evaluate performance data

»Respond to outcome measurement findings

»Comply with documentation requirements

2. Customer and patient engagement

"Assess customer and patient satisfaction

»Develop strategies to address satisfaction issues

3. Patient safety

»Monitor and report sentinel events

»Participate in root cause analysis

»Promote evidence-based practices

»Manage incident reporting

4. Maintain survey and regulatory readiness

5. Monitor and promote workplace safety requirements

6. Promote intra/interdepartmental communication

\section{FOUNDATIONAL THINKING SKILLS}

1. Apply systems thinking knowledge as an approach to analysis and decision-making

2. Understand complex adaptive systems definitions and applications 


\section{E. TECHNOLOGY}

1. Information technology-Understand the effect of IT on patient care and delivery systems to reduce work load

»Ability to integrate technology into patient care processes

» Use information systems to support business decisions

\section{F. STRATEGIC MANAGEMENT}

1. Facilitate change

»Assess readiness for change

"Involve staff in change processes

»Communicate changes

»Evaluate outcomes

2. Project management

» Identify roles

»Establish timelines and milestones

»Allocate resources

»Manage project plans

3. Contingency plans

» Manage internal disaster or emergency planning and execution

» Manage external disaster or emergency planning and execution

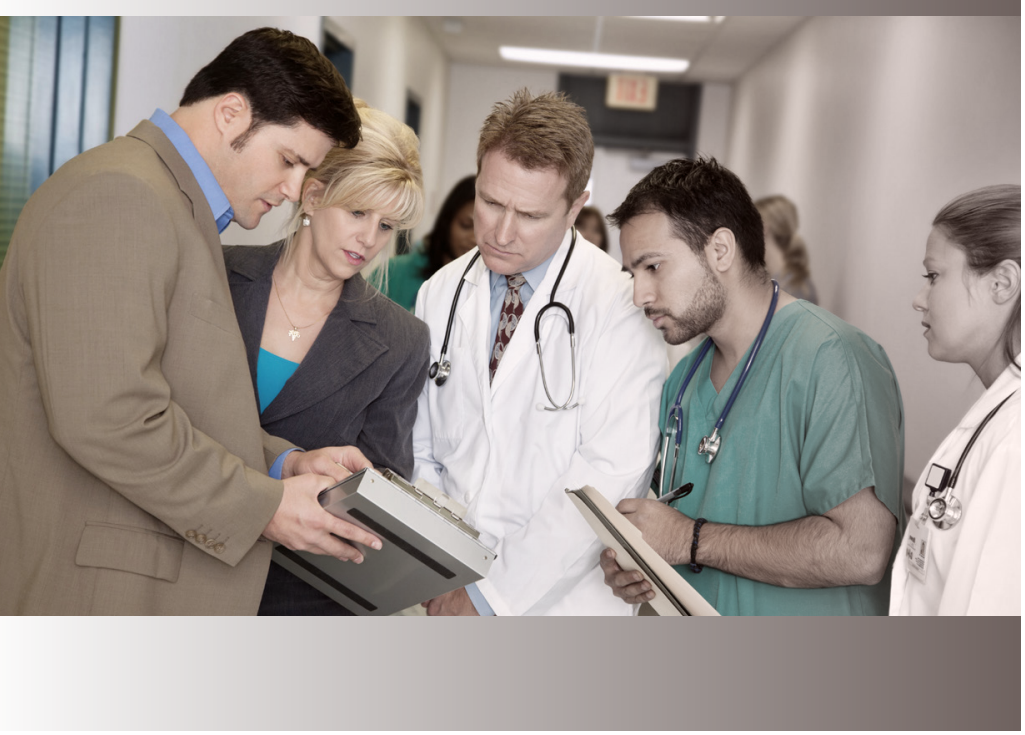

4. Demonstrate written and oral presentation skills

5. Manage meetings effectively

6. Demonstrate negotiation skills

7. Influence the practice of nursing through participation in professional organizations

8. Collaborate with other service lines

9. Shared decision-making

» Establish vision statement

» Facilitate a structure of shared governance

"Implement structures and processes

"Support a just culture

10. Support a culture of innovation

G. APPROPRIATE CLINICAL PRACTICE KNOWLEDGE

(Determined by specific role and institution)

1. Each role and institution has expectations regarding the clinical knowledge and skill required of the role. These expectations should be established for the specific individual based on organizational requirements. 


\section{THE ART}

A. HUMAN RESOURCE LEADERSHIP SKILLS

1. Performance management

»Conduct staff evaluations

» Assist staff with goal-setting

»Implement continual performance development

»Monitor staff for fitness for duty

"Initiate corrective actions

» Terminate staff

2. Staff development

» Facilitate staff education and needs assessment

»Ensure competency validation

»Promote professional development of staff

» Facilitate leadership growth among staff

"Identify and develop staff as part of a succession planning program

3. Staff retention

»Assess staff satisfaction

»Develop and implement strategies to address satisfaction issues

»Promote retention

» Develop methods to reward and recognize staff

\section{B. RELATIONSHIP MANAGEMENT AND INFLUENCING BEHAVIORS}

1. Manage conflict

2. Situation management

»Identify issues that require immediate attention

» Apply principles of crisis management to handle situations as necessary
3. Relationship management

»Promote team dynamics

»Mentor and coach staff and colleagues

»Apply communication principles

4. Influence others

»Encourage participation in professional action

» Role model professional behavior

" Apply motivational theory

»Act as change agent

»Assist others in developing problem solving skills

» Foster a healthy work environment

5. Promote professional development

»Promote stress management

"Apply principles of self-awareness

»Encourage evidence-based practice

»Apply leadership theory to practice

\section{DIVERSITY}

1. Cultural competence

» Understand the components of cultural competence as they apply to the workforce

2. Social justice

»Maintain an environment of fairness and processes to support it

3. Generational diversity

»Capitalize on differences to foster highly effective work groups 
A. PERSONAL AND PROFESSIONAL ACCOUNTABILITY

1. Personal growth and development

»Manage through education advancement, continuing education, career planning and annual selfassessment and action plans

2. Practice ethical behavior

»Including practice that supports nursing standards and scopes of practice

3. Involvement in professional associations

»Including membership and involvement in an appropriate professional association that facilitates networking and professional development

4. Achieve certification in an appropriate field/specialty

\section{B. CAREER PLANNING}

1. Know your role

» Understand current job description/ requirements and compare those to current level of practice

2. Know your future

»Plan a career path

3. Position yourself

»Develop a of career path/plan that provides direction while offering flexibility and capacity to adapt to future scenarios
C. PERSONAL JOURNEY DISCIPLINES

1. Apply action learning

»Apply techniques of "action learning" to problem solve and personally reflect on decisions

2. Engage in reflective practice

"Includes knowledge of, and active practice of reflection as a leadership behavior

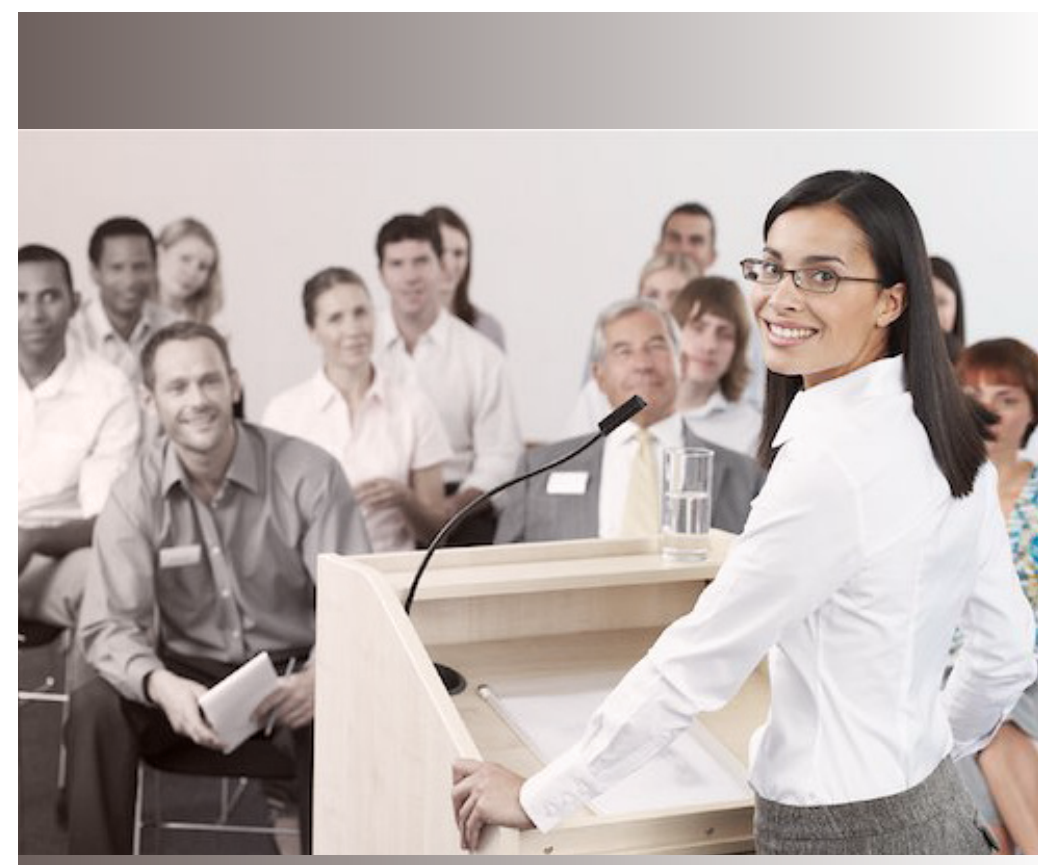




\section{REFLECTIVE PRACTICE REFERENCE BEHAVIORS/TENETS}

Utilizing a set of guidelines and tenants that facilitate reflective practice; these may be individually developed or can be based on specific models developed by others; below are the "Dimensions of Leadership" developed by the Center for Nursing Leadership, which offer an example of a set of guidelines/tenants that can be used as a tool to guide personal reflection of an individual's leadership behaviors.

\section{Holding the truth}

The presence of integrity as a key value of leadership

\section{Appreciation of ambiguity}

Learning to function comfortably amid the ambiguity of our environments

3. Diversity as a vehicle to wholeness

The appreciation of diversity in all its forms: race, gender, religion, sexual orientation, generational, the dissenting voice and differences of all kinds

\section{Holding multiple perspectives without judgment}

Creation and holding a space so that multiple perspectives are entertained before decisions are rendered

\section{Discovery of potential}

The ability to search for and find the potential in ourselves and in others

\section{Quest for adventure towards knowing}

Creating a constant state of learning for the self, as well as an organization

\section{Knowing something of life}

The use of reflective learning and translation of that learning to the work at hand

\section{Nurturing the intellectual and emotional self}

Constantly increasing one's knowledge of the world and the development of the emotional self

\section{Keeping commitments to oneself}

Creating the balance that regenerates and renews the spirit and body so that it can continue to grow 